\title{
The Relevance of Breast Cancer Subtypes in the Outcome of Neoadjuvant Chemotherapy
}

\author{
M. E. Straver, MD ${ }^{1}$, E. J. Th. Rutgers, MD, $\mathrm{PhD}^{1}$, S. Rodenhuis, MD, $\mathrm{PhD}^{2}$, S. C. Linn, MD, $\mathrm{PhD}^{2}$, C. E. Loo, $\mathrm{MD}^{3}$, \\ J. Wesseling, MD, $\mathrm{PhD}^{4}$, N. S. Russell, MD, $\mathrm{PhD}^{5}$, H. S. A. Oldenburg, MD, $\mathrm{PhD}^{1}$, N. Antonini, Msc ${ }^{6}$, \\ and M. T. F. D. Vrancken Peeters, MD, PhD ${ }^{1}$ \\ ${ }^{1}$ Department of Surgical Oncology, Netherlands Cancer Institute-Antoni van Leeuwenhoek Hospital, Amsterdam, \\ The Netherlands; ${ }^{2}$ Department of Medical Oncology, Netherlands Cancer Institute-Antoni van Leeuwenhoek Hospital, \\ Amsterdam, The Netherlands; ${ }^{3}$ Department of Radiology, Netherlands Cancer Institute-Antoni van Leeuwenhoek \\ Hospital, Amsterdam, The Netherlands; ${ }^{4}$ Department of Pathology, Netherlands Cancer Institute-Antoni van \\ Leeuwenhoek Hospital, Amsterdam, The Netherlands; ${ }^{5}$ Department of Radiotherapy, Netherlands Cancer Institute- \\ Antoni van Leeuwenhoek Hospital, Amsterdam, The Netherlands; ${ }^{6}$ Department of Biostatistics, Netherlands Cancer \\ Institute-Antoni van Leeuwenhoek Hospital, Amsterdam, The Netherlands
}

\begin{abstract}
Background. Breast cancer is increasingly considered a heterogeneous disease. The aim of this study was to assess the differences between histological and receptor-based subtypes in breast-conserving surgery and pathological complete response (pCR) after neoadjuvant chemotherapy. Method. A consecutive series of 254 patients with operable breast cancer treated with neoadjuvant chemotherapy was analyzed. Tumors were classified according to their receptor status in estrogen receptor (ER)-positive tumors (HER2-negative), triple-negative tumors, and HER2-positive tumors. The type of surgery feasible prior to neoadjuvant chemotherapy was compared with the actual surgery performed.

Results. The overall increase in breast-conserving surgery was $37 \%$ (73 of 198). In patients with ductal and lobular carcinomas this increase was $41 \%$ (63 of $152,95 \%$ confidence interval [95\% CI] $0.34-0.49)$ and $20 \%$ (7 of $35,95 \%$ CI 0.10-0.36), respectively $(P=0.02)$. Half of the patients with lobular carcinoma had to undergo a secondary mastectomy because of incomplete resection margins. In ERpositive, triple-negative and HER2-positive tumors, the increase in breast-conserving surgery was 39\% (42 of 109, $95 \%$ CI $0.30-0.48$ ), $24 \%$ (11 of $45,95 \%$ CI $0.14-0.38$ ),
\end{abstract}

(C) The Author(s) 2010. This article is published with open access at Springerlink.com

First Received: 19 July 2009;

Published Online: 6 April 2010

M. E. Straver, MD

e-mail:m.straver@nki.nl and $45 \%$ (20 of $44,95 \%$ CI $0.32-0.60)(P=0.11)$. The pCR rate in ductal and lobular carcinomas was $12 \%$ (23 of $195)$ and $2 \%(1$ of 42$)$, respectively $(P=0.09)$. In ERpositive, triple-negative and HER2-positive tumors the pCR rates were $2 \%$ ( 3 of 138), $28 \%$ (16 of 57 ), and $18 \%$ (10 of 56), respectively. Multivariate analysis showed that the receptor-based subtype was the only significant predictor of pCR $(P=0.004)$.

Conclusion. In lobular tumors the benefit with regard to breast-conserving surgery of neoadjuvant chemotherapy is questionable. Although in ER-positive tumors the pCR rate is low, the increase in breast-conserving surgery was remarkable in ductal ER-positive tumors.

Neoadjuvant chemotherapy is the standard of care in patients with locally advanced breast cancer and is increasingly being used in the treatment of patients with large operable breast cancer or proven lymph node metastases. In theory, early eradication of micrometastases would prevent the outgrowth of distant metastases and herewith improve survival. However, an overall survival benefit for neoadjuvant chemotherapy compared with adjuvant chemotherapy has yet to be proven. ${ }^{1}$ The use of neoadjuvant chemotherapy in operable breast cancer has other advantages. Firstly, downstaging of the tumor load increases the rate of breast-conserving surgery. ${ }^{2}$ Secondly, neoadjuvant chemotherapy creates the opportunity to assess in vivo the tumor response without requiring longterm follow-up since pathological complete remission of the primary tumor is associated with an improved 
survival. ${ }^{1,3}$ Furthermore, response monitoring during the chemotherapy course may potentially create the ability to adjust the chemotherapy regimen to the response of the primary tumor at an early stage. ${ }^{4}$ Tailored treatment could be a major gain of neoadjuvant treatment and might ultimately lead to a survival benefit. However, the first trial addressing this question, the Gepartrio trial, showed no benefit of switching nonresponders. ${ }^{5}$

Neoadjuvant chemotherapy offers the unique opportunity to assess the sensitivity of an individual tumor to chemotherapy in the context of different histological and pathological variables. This is in particular helpful since breast cancer is increasingly recognized as a heterogeneous disease and the need for individualized therapy is widely accepted. Major biological and clinical differences exist between the two most common histological subtypes; invasive ductal carcinoma and invasive lobular carcinoma. Invasive ductal carcinoma is the most common type of breast cancer and originates from the milk ducts. Invasive lobular carcinoma starting in the milk-producing lobules is characterized by a diffuse grown pattern (the so-called Indian-file pattern) and the loss of E-cadherine expression (a glycoprotein that mediates adhesion between epithelial cells). Lobular carcinoma is more frequently positive for the estrogen receptor than ductal carcinoma. At presentation, patients with lobular disease are typically older and their tumors tend to be larger in size. Unfortunately, almost none of the randomized trial assessing chemosensitivity in the adjuvant or the neoadjuvant setting report the effectiveness of chemotherapy separately for patients with lobular carcinoma. ${ }^{6}$ Six retrospective case series of neoadjuvant chemotherapy showed a pathological complete response (pCR) rate in lobular carcinoma of $1.7 \%$ (6 of 354) compared with $11.6 \%$ (300 of 2,584) in ductal carcinoma. $^{7-9}$ Despite the low pCR rate, patients with lobular carcinoma had a higher survival compared with patients with more chemosensitive ductal tumors. ${ }^{8,9}$

Besides the histological subtypes, gene-expression profiling has led to the identification of five different molecular breast cancer subtypes (i.e., luminal A, luminal $\mathrm{B}$, basal and HER2 subtype, and normal-like subtypes). ${ }^{10,11}$ To a degree, these molecular subtypes can be also be distinguished using immunohistochemistry. ${ }^{12}$ Subtyping of breast cancer by immunohistochemistry assays for estrogen receptor (ER), progesterone receptor (PR), and human epidermal growth factor 2 (HER2), yields three broad groups: (1) ER-positive tumors, when ER is positive and HER2 is not amplified; (2) triple-negative tumors when ER, PR, and HER2 are all negative, and (3) HER2-positive tumors, which may be ER-positive or ER-negative; The molecular subtypes have been associated with prognosis. The luminal A subtype has been shown to have a better prognosis compared with the other subtypes and the basal subgroup has the worst prognosis. ${ }^{10,11,13}$ Comparably, triple-negative tumors have a worse prognosis than ERpositive tumors. ${ }^{14}$ Research increasingly suggests that the molecular subtypes are associated with chemosensitivity. The basal and triple-negative subtypes has been shown to be more sensitive to chemotherapy than the luminal and ER-positive breast cancer. ${ }^{15-18}$

The aim of this manuscript was to analyze differences in the outcome of neoadjuvant chemotherapy between both histological and receptor-based subtypes. Primary outcome measures were the increase in breast-conserving therapy and pathological complete remission.

\section{METHODS}

\section{Patients}

A consecutive series of 254 patients with primary operable breast tumors who received neoadjuvant chemotherapy between January 2000 and April 2007 were retrospectively analyzed. In our institute, patients with invasive breast cancer greater than $3 \mathrm{~cm}$ and/or involved lymph nodes are typically eligible for treatment within a neoadjuvant chemotherapy protocol. Patients with inoperable breast tumors (T4 and/or N3) were excluded from this analysis. Patients received neoadjuvant chemotherapy in 1 of 2 clinical studies ongoing or received treatment according to the standard arm of these trials. ${ }^{19}$ The clinical studies were approved by the institutional ethical committee and informed consent was obtained from all patients.

The tumor size was assessed with ultrasound, mammography, and contrast-enhanced MRI.

Nodal status prior to neoadjuvant chemotherapy was determined by ultrasound-guided fine-needle aspiration or, when negative, sentinel node biopsy. Prior to neoadjuvant treatment, 14-gauge biopsies of the breast tumor were taken under ultrasound guidance to determine the histological subtype, hormone receptor, and HER2-status. In the majority of patients a marker was placed in the tumor for surgical detection and for optimal pathological examination of the tumor area in the surgical specimen after neoadjuvant chemotherapy.

\section{Chemotherapy Regimens}

Since the year 2000, patients could participate in a study randomizing patients between AC ( 6 cycles of doxorubicin $60 \mathrm{mg} / \mathrm{m}^{2}$ and cyclophosphamide $600 \mathrm{mg} / \mathrm{m}^{2}$, every 3 weeks) and AD (6 cycles of doxorubicin $50 \mathrm{mg} / \mathrm{m}^{2}$ and docetaxel $75 \mathrm{mg} / \mathrm{m}^{2}$ ). A total of 107 patients were treated in this period; 51 participated in the trial, and 56 were 
treated according to the standard arm (AC). In 2005 this trial was followed by a new neoadjuvant trial. In this trial the treatment regimen depended on the presence or absence of HER2 amplification. Furthermore, the tumor response was evaluated by contrast-enhanced MRI and clinical examination after 1 or 3 cycles (depending on the specific regimen). ${ }^{4}$ Chemotherapy regimens were changed to a hypothetically non-cross-resistant regimen when failure of response was apparent upon radiological evaluation. Preoperative chemotherapy for HER2-negative tumors employed 1 of the following regimens: dose dense (dd) AC (AC every 2 weeks with filgrastim) and/or DC (6 cycles of docetaxel $75 \mathrm{mg} / \mathrm{m}^{2}$ and capecitabine $2 \times \mathrm{dd}$ $1,000 \mathrm{mg} / \mathrm{m}^{2}$ orally during 14 days, every 3 weeks). ${ }^{20-22}$ For HER2-positive tumors, the regimens included PTC (paclitaxel $80 \mathrm{mg} / \mathrm{m}^{2} /$ week, trastuzumab $2 \mathrm{mg} / \mathrm{kg}$, and carboplatin AUC 2 to $3 \mathrm{mg} / \mathrm{ml}$ per minute, given weekly times 6 , followed by 2 weeks trastuzumab alone, for 3 cycles). Of the 144 patients treated after 2005, 63 patients participated in this trial and 81 patients were treated according the standard arm of the protocol, with ddAC chemotherapy.

\section{Surgical and Adjuvant Treatment}

The type of surgical treatment, breast-conserving surgery or mastectomy, feasible at the time of diagnosis was retrospectively assessed by an experienced breast surgeon (MVP). The type of surgery feasible prior to neoadjuvant chemotherapy was compared with the actual surgery performed after neoadjuvant chemotherapy. Recommendation for surgery was made by a multidisciplinary team of breast cancer specialists including surgeons, radiologists, pathologists, radiation, and medical oncologists. Treatment decisions were mainly based on patient's desires and surgical considerations involving the breast-tumor index, age, multifocality, localization, histology, and the presence of ductal invasive carcinoma in situ (DCIS). All patients with proven axillary lymph node metastases prior to neoadjuvant chemotherapy underwent an axillary lymph node dissection (ALND) at levels I and II with level III sampling after neoadjuvant chemotherapy. Level III sampling was done to stage for adjuvant radiotherapy indications. ${ }^{23}$ Patients undergoing breast-conserving surgery received radiation to the breast with a boost to the tumor bed. The indication for locoregional radiation therapy (chest wall and regional nodal basins) was based on the original staging. Hormone receptor-positive patients received adjuvant endocrine treatment for at least 5 years and HER2-positive patients received trastuzumab for 1 year.

\section{Pathological Examination}

Tumors were classified according to the standard criteria of the World Health Organization. Estrogen receptor (ER) status and progesterone receptor status were determined by immunohistochemistry and interpreted positive if more than $10 \%$ of the nuclei stained positive. HER 2 status was assessed by scoring the intensity of membrane staining using immunohistochemistry. Tumors with a score of $3+$ (strong homogeneous staining) were considered HER2positive. In case of $2+$ scores (moderate homogeneous staining) chromogenic in situ hybridization (CISH) was used to determine amplification. The tumor margins were defined as a clear pathologic margin if the distance was $>2 \mathrm{~mm}$ on microscopic evaluation. Pathological complete response (pCR) was defined as the absence of invasive carcinoma in both the breast and axilla at microscopic examination of the resection specimen, regardless of the presence of carcinoma in situ. ${ }^{24}$

\section{Statistical Analysis}

Analyses were performed using SPSS version 15.1 (SPSS Inc, Chicago, IL) and SAS version 9.1 (SAS Institute Inc. Cary, NC, VS) Differences in clinical data were tested using the $\chi^{2}$ or Fisher exact test where appropriate. A multivariate logistic regression model was built to examine the associations between pCR and age, menopausal status, tumor stage (T1 vs. T2 vs. T3), $\mathrm{N}$ stage (N0 vs. N1), molecular subtype based on tumor receptor status (ERpositive vs. triple-negative vsHER2-positive), histology (ductal vs. lobular vs. other). The level of significance was set at 0.05 .

\section{RESULTS}

Table 1 shows the patient and tumor characteristics. The median age was 45 years (range 23-76), and the majority of patients were premenopausal $(70 \%)$ and had ductal carcinomas $(78 \%)$. Also, $70 \%$ of the patients were treated with an antracycline-based regimen (AC), $20 \%$ with a taxane-based regimen (AD or $\mathrm{CD}$ ), and $8 \%$ received trastuzumab in combination with chemotherapy (PTC).

\section{Increase in Breast-Conserving Surgery}

After neoadjuvant chemotherapy, 251 of 254 patients received surgery to the breast; three patients refused additional surgical treatment. Before the administration of neoadjuvant chemotherapy, 53 patients could be treated with breast-conserving therapy whereas mastectomy was indicated in the remaining 198 patients. After neoadjuvant 
TABLE 1 Patient and tumor characteristics

\begin{tabular}{|c|c|c|}
\hline & No. & $\%$ \\
\hline Total number of patients & 254 & \\
\hline Median age (range) & $45(23-76)$ & \\
\hline \multicolumn{3}{|l|}{ Menopausal status } \\
\hline Premenopausal & 179 & 70 \\
\hline Perimenopausal & 8 & \\
\hline Postmenopausal & 59 & 23 \\
\hline Unknown & 8 & \\
\hline \multicolumn{3}{|l|}{ T-stage } \\
\hline $\mathrm{T} 1$ & 13 & \\
\hline $\mathrm{T} 2$ & 151 & 59 \\
\hline $\mathrm{T} 3$ & 90 & 35 \\
\hline \multicolumn{3}{|l|}{$\mathrm{N}$-stage } \\
\hline N0 $\left(\mathrm{SNB}^{-}\right)$ & 43 & 17 \\
\hline $\mathrm{N} 1\left(\mathrm{FNA}^{+} / \mathrm{SNB}^{+}\right)$ & 170 & 67 \\
\hline NX & 41 & 16 \\
\hline \multicolumn{3}{|l|}{ Histology } \\
\hline Ductal & 197 & 78 \\
\hline Lobular & 37 & 15 \\
\hline Mixed & 6 & \\
\hline Adenocarcinoma (NS) & 14 & \\
\hline \multicolumn{3}{|l|}{ Receptor-based subtype } \\
\hline ER-positive $\left(\mathrm{ER}^{+} ; \mathrm{HER} 2^{-}\right)$ & 140 & 55 \\
\hline Triple-negative $\left(\mathrm{ER}^{-} ; \mathrm{PR}^{-} ; \mathrm{HER} 2^{-}\right)$ & 58 & 23 \\
\hline $\mathrm{HER} 2^{+}$ & 56 & 22 \\
\hline \multicolumn{3}{|l|}{ Participating in trial } \\
\hline Study 1 (before 2005 , without switch) & 51 & 20 \\
\hline Without randomization & 56 & 22 \\
\hline Study 2(after 2005, with switch) & 63 & 45 \\
\hline Without randomization & 81 & 32 \\
\hline Other & 3 & 1 \\
\hline \multicolumn{3}{|l|}{ Chemotherapy regimen } \\
\hline Doxorubicin-cyclophosphamide (AC) & 178 & 70 \\
\hline Capecitabine-docetaxel (CD) & 24 & 10 \\
\hline Paclitaxel-trastuzumab-carboplatin (PTC) & 20 & 8 \\
\hline Doxorubicin-docetaxel (AD) & 26 & 10 \\
\hline Other & 6 & 2 \\
\hline \multicolumn{3}{|l|}{ Surgery } \\
\hline Yes & 25 & 98 \\
\hline No & 2 & 1 \\
\hline Axillary lymph node dissection only & 1 & 1 \\
\hline
\end{tabular}

SNB sentinel node biopsy, FNA fine needle aspiration, ER estrogenreceptor, $P R$ progesterone receptor, $H E R 2$ human epidermal growth factor receptor $2, N S$ not specified

chemotherapy, breast-conserving surgery was possible in an additional 73 patients. Thus, the increase in breastconserving surgery was $37 \%$ (73 of 198). None of the 53 patients, in whom breast-conserving surgery was feasible prior to neoadjuvant chemotherapy, had to undergo a mastectomy after neoadjuvant chemotherapy because of progressive disease.

The tumor margin was not clear after breast conserving therapy (including carcinoma in situ) in 32 of 135 patients (24\%). In 21 patients no re-excision or subsequent mastectomy was done because the tumor was only marginally incomplete excised. These patients were treated with adjuvant radiotherapy. Two patients were treated with a reexcision. In nine patients, the incomplete resection margins led to a subsequent mastectomy, due to more than focally involved margins, and no room for further local resections to achieve a cosmetically acceptable outcome.

After a median follow up of 35 months (range, 5-101), eight patients $(3 \%, 8$ of 251$)$ had a local recurrence. The local recurrence rate was similar in the group of patients that were switched to breast-conserving surgery (3\%, 2 of 73). The median overall survival and disease-free survival were $90 \%$ (95\% confidence interval [95\% CI] 86\%-94\%) and $86 \%$ (95\% CI 82\%-91\%), respectively.

Figure 1 shows the findings at surgery separately for ductal and lobular carcinomas. Of the patients with ductal carcinomas ( $n=195$ ), 152 patients initially had an indication for mastectomy. After neoadjuvant chemotherapy, 65 patients underwent breast-conserving surgery. Two patients had incomplete resection margins, leading to a subsequent mastectomy. Thus, the increase in breast conserving surgery was $41 \%$ (63 of 152). Of the patients with lobular carcinomas $(n=42), 35$ patients initially had an indication for mastectomy; 14 of these patients underwent breast-conserving surgery after neoadjuvant chemotherapy. Unfortunately, 7 of the 14 patients $(50 \%)$ had to subsequently undergo a mastectomy due to incomplete resection margins. The increase in breast-conserving surgery in lobular carcinoma was $20 \%$ (7 of 35) and was significantly lower than in ductal carcinoma $(P=0.02)$.

Figure 2 shows the findings at surgery separately for the different subtypes based on receptor status. Of the patients with ER-positive tumors $(n=138), 109$ patients initially had an indication for mastectomy. After neoadjuvant chemotherapy 50 patients underwent breast-conserving surgery. Eight patients $(16 \%)$ had incomplete resection margins leading to a subsequent mastectomy. Thus, the increase in breast-conserving surgery was 39\% (42 of 109). Remarkably, seven of the eight patients in the ER-positive group with incomplete resection margins leading to a secondary mastectomy had a lobular carcinoma.

Of the patients with triple-negative tumors $(n=57), 45$ patients initially had an indication for mastectomy. The increase in breast-conserving surgery was $24 \%$ (11 of 45), and none of the patients had incomplete resection margins leading to a secondary mastectomy. In the patients with HER2-positive tumors $(n=56)$ a mastectomy was indicated before neoadjuvant chemotherapy in 44 patients. The 
FIG. 1 Increase in breastconserving surgery by histological subtype. The type of surgery feasible prior to neoadjuvant chemotherapy is compared with the actual surgery performed after neoadjuvant chemotherapy. The increase in breast-conserving surgery (shown in the blue boxes) is higher in ductal carcinomas. NAC, neoadjuvant chemotherapy; BCS, breast-conserving surgery

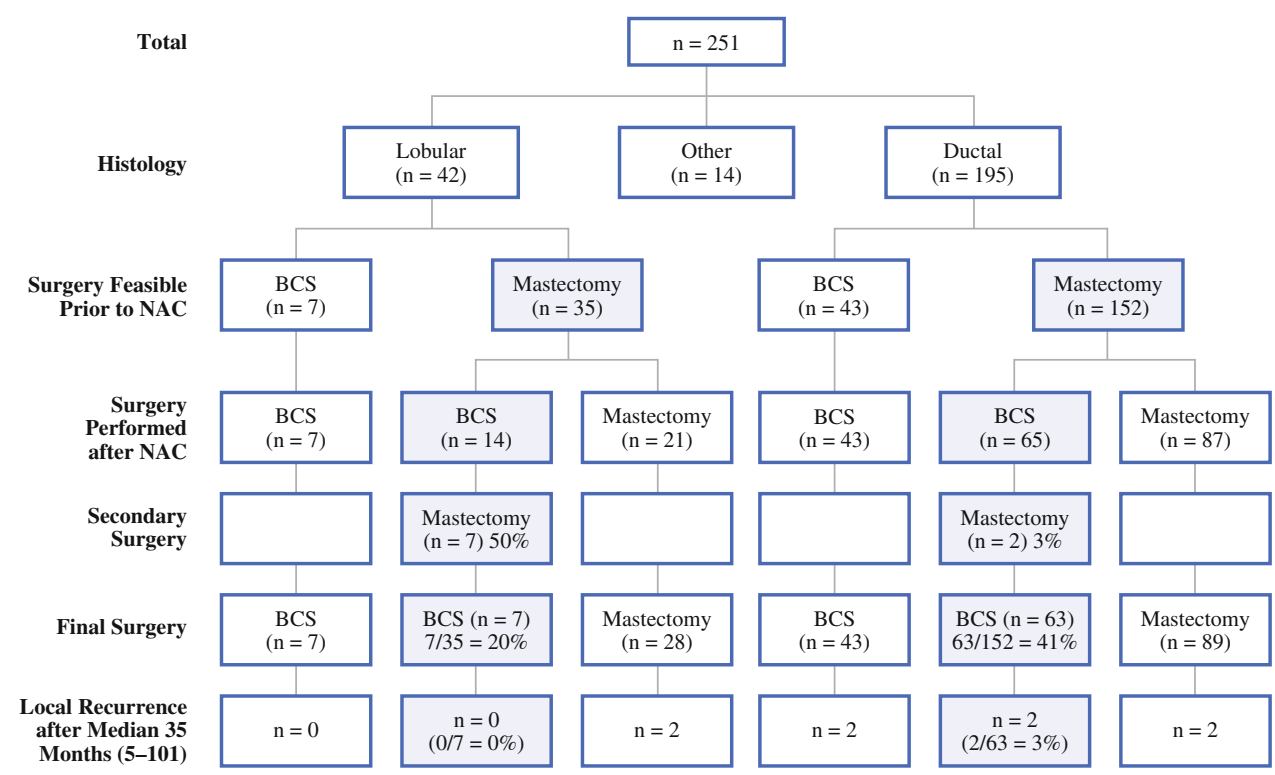

FIG. 2 Increase in breastconserving surgery by receptorbased subtype. The type of surgery feasible prior to neoadjuvant chemotherapy is compared with the actual surgery performed after neoadjuvant chemotherapy. The increase in breast-conserving surgery (shown in the blue boxes) is highest in HER2-positive carcinomas. NAC, neoadjuvant chemotherapy; BCS, breast conserving surgery



increase in breast-conserving surgery was $45 \%$ (20 of 44), and in one patient (5\%) a secondary mastectomy was performed due to incomplete resection margins. The increase in breast-conserving surgery was not significantly different between the receptor based subtypes $(P=0.11)$.

\section{Pathological Complete Response ( $p C R$ ) Rates}

Overall, 12\% (29 of 251) of the patients who had surgery after neoadjuvant chemotherapy achieved a pCR of both the primary tumor and lymph node metastases. A complete remission of only the primary tumor in the breast was seen in $18 \%$ ( 45 of 251 ) (Table 2). The axillary pCR rate was calculated from the group of patients with proven lymph node metastases prior to neoadjuvant chemotherapy, in whom the positive lymph nodes were left in situ. Thus, the patients with a positive sentinel node biopsy prior to neoadjuvant chemotherapy were excluded $(n=24)$. The axillary pCR rate was $16 \%$ (23 of 146) in this group of patients.

Patients with ductal carcinoma had a higher pCR rate compared with patients with lobular carcinoma, $12 \%$ (23 of $195)$ vs. $2 \%$ ( 1 of 42$)$, respectively $(P=0.09)$. Based on the receptor status, the pCR rate in ER-positive, triplenegative, and HER2-positive tumors was $2 \%$ (3 of 138), $28 \%$ (16 of 57), and 18\% (10 of 56), respectively $(P<0.001)$. HER2-positive patients treated with trastuzumab and chemotherapy achieved a pCR in $35 \%$ (7 of 
TABLE 2 Pathological response according to histological and molecular subtype

\begin{tabular}{lrcc}
\hline & No. & $\begin{array}{l}\text { pCR breast } \\
\text { and axilla } \\
\text { No. }(\%)\end{array}$ & $\begin{array}{l}\text { pCR } \\
\text { breast } \\
\text { No. }(\%)\end{array}$ \\
\hline $\begin{array}{l}\text { Histological subtype } \\
\text { Ductal }\end{array}$ & 195 & $23(12)$ & $36(18)$ \\
Lobular & 42 & $1(2)$ & $1(2)$ \\
$\quad \begin{array}{l}\text { Adenocarcinoma (NS) } \\
\text { Receptor-based subtype }\end{array}$ & 14 & $5(36)$ & $8(57)$ \\
$\begin{array}{l}\text { ER-positive } \\
\quad \text { HER2-negative) }\end{array}$ & 138 & $3(2)$ & $9(7)$ \\
$\quad \begin{array}{l}\text { Triple-negative } \\
\text { HER2+ }\end{array}$ & 57 & $16(28)$ & $20(35)$ \\
$\quad$ HER2+T & 36 & $3(8)$ & $5(14)$ \\
Total & 20 & $7(35)$ & $11(55)$ \\
\hline
\end{tabular}

pCR pathological complete remission, ER estrogen receptor, HER2 human epidermal growth factor receptor 2, Triple-negative ER and progesterone receptor (PR) and HER2-negative (triple negative), $H E R 2+T$ HER2-positive and treated with trastuzumab

20) compared with $8 \%$ (3 of 36) without trastuzumab $(P=0.025)$. Additionally, we assessed variables that were predictive for a $\mathrm{pCR}$ in a multivariate analysis. Among the variables age, menopausal status, tumor stage, $\mathrm{N}$ stage, and histology, the receptor-based subtype was the only significant predictor of $\mathrm{pCR}(P=0.004)$, with triple-negative patients being more chemosensitive (odds ratio $14.8,95 \%$ CI 2.79-78.4) than ER-positive tumors. With regard to the response of the primary tumor regardless of the axillary response, multivariate analysis showed that both T-stage $(P=0.006)$ and receptor-based subtype $(P=0.002)$ significantly predicted a complete remission.

\section{DISCUSSION}

In this study, we assessed the outcome of neoadjuvant chemotherapy in different breast cancer subtypes in a consecutive series of 254 patients with operable breast cancer.

In lobular carcinomas, the pathological response rate as well as the increase in breast conserving was relatively low, $2 \%$ and $20 \%$, respectively. Moreover, in half of the patients a secondary mastectomy had to be performed due to incomplete resection margins. This might be explained by the difficulty of determining the size of residual disease after neoadjuvant chemotherapy in lobular carcinoma patients even when using contrast enhanced MRI. ${ }^{25,26}$ Their unique linear and scattered growth pattern may account for these problems. Reducing the vascularity might appear as a decrease in tumor size on MRI, while it does not influence the malignant cells growing single file, resulting in underestimation of the tumor size. Further research focusing on the imaging of lobular carcinomas after neoadjuvant chemotherapy is warranted. Based on our results we recommend informing patients with lobular disease about the risk on a secondary mastectomy and to be cautious in offering these patients breast-conserving surgery after neoadjuvant chemotherapy. Moreover, the problem in surgical treatment suggests that the majority of patients with lobular breast cancer have no clinical benefit of neoadjuvant treatment above adjuvant chemotherapy. Therefore, it might be argued that direct surgical treatment should be considered outside clinical studies. Several studies examining the differences between lobular and ductal carcinomas show a low pCR rate of $0-3 \%$ in patients with lobular carcinomas. ${ }^{6,9,27}$ The only patient with a lobular carcinoma achieving a pCR in our series had a HER2-positive tumor and was treated with trastuzumab and chemotherapy. This finding suggests that receptorstatus is very predictive for chemosensitivity. In addition, this study shows that the receptor-based subtype was the only variable significantly associated with $\mathrm{pCR}$ in the multivariate analysis. ER-positive tumors had lower pCR rate compared with triple-negative and HER2-positive breast cancer. Carey et al. observed similar results when classifying patients in molecular subgroups according to immunohistochemical staining. ${ }^{15}$ Remarkably, the increase in breast-conserving surgery was high in ER-positive ductal tumors (39\%), which implies that the tumor load reduces significantly. This finding suggests that treatment with neoadjuvant chemotherapy does have a clinical benefit in a large number of patients with ER-positive disease $(39 \%)$. It is questionable whether pathological complete response is the best surrogate marker for the chemosensitivity in estrogen receptor-positive breast cancer. The low incidence of $\mathrm{pCR}$ hampers research to predictive factors of chemosensitivity and the comparison of different treatment regimens in the relatively large group of estrogen receptorpositive disease. ${ }^{28}$ Recently a new surrogate, the residual cancer burden (RCB), has been proposed to measure chemosensitivity as a continuous variable derived from the primary tumor dimensions, cellularity of the tumor bed, and axillary nodal burden. ${ }^{29} \mathrm{RCB}$ was a significant predictor of distant relapse-free survival and can be used to more precisely determine the chemosensitivity.

HER2-positive patients showed a both a high pCR rate $(18 \%)$ as well as a significant increase in breast-conserving surgery (45\%). HER2 amplification is observed in 15-25\% of the patients with breast cancer, especially in ductal carcinomas. The benefit of adding trastuzumab to neoadjuvant regimens, as seen in this report, has been proven in a randomized trial showing a pCR rate of $65 \%$ in HER2positive patients treated chemotherapy combined with trastuzumab compared to a pCR rate of $26 \%$ in patients treated with chemotherapy only. ${ }^{30}$ In our study we 
classified all HER2-positive tumors into 1 subgroup. However, HER2-positive tumors that are ER-positive are less chemosensitive than HER2-positive tumors that are ER-negative. In this report we did not subdivide the HER2positive patients because of the small sample size and different treatment regimens within this subgroup.

Triple-negative tumors showed a high pCR rate $(28 \%)$ and a slightly lower increase in breast conserving surgery (24\%) compared with the other subtypes. This might be explained by the high incidence of BRCA carriers (7 of 58 patients in this series) and young patients, in which a mastectomy is preferably performed or desired.

The overall increase in breast-conserving surgery assessed in our patient population with operable breast cancer was high and the local recurrence is low after a median follow-up of 34 months. Several aspects might have contributed to this high success rate. Firstly, marking of the primary tumor prior to neoadjuvant chemotherapy is important. After neoadjuvant chemotherapy, many of the tumors may become nonpalpable and difficult to visualize by imaging modalities. The marker can be used to localize the tumor before surgery and assists the pathologist in detecting the tumor-bearing area. Secondly, evaluation of the residual disease with contrast enhanced MRI is superior to conventional methods. ${ }^{31,32}$ The ability of MRI to distinguish fibrous from vascularized tissue after the administration of contrast medium underlies this advantage. However, the negative predictive value of MRI is low. ${ }^{25}$ Therefore, we never omit surgical treatment in complete responders assessed at MRI.

In conclusion, this study shows that it is essential to obtain information about the histology and the hormone and HER2 receptor status before the administration of neoadjuvant chemotherapy. Patients with lobular carcinomas should be well informed about the low probability of having a pCR and breast-conserving surgery with complete resection margins after neoadjuvant chemotherapy. Patients with estrogen receptor positive disease have a low likelihood of achieving a pCR; however, the increase in breast-conserving therapy is remarkable in ductal carcinomas. Furthermore, the breast cancer subtype will become increasingly important to determine the type of regimen as currently applied in HER2-positive patients.

OPEN ACCESS This article is distributed under the terms of the Creative Commons Attribution Noncommercial License which permits any noncommercial use, distribution, and reproduction in any medium, provided the original author(s) and source are credited.

\section{REFERENCES}

1. Rastogi P, Anderson SJ, Bear HD, Geyer CE, Kahlenberg MS, Robidoux A, et al. Preoperative chemotherapy: updates of
National Surgical Adjuvant Breast and Bowel Project Protocols B-18 and B-27. J Clin Oncol. 2008;26:778-85.

2. Mieog JS, van der Hage JA, van de Velde CJ. Neoadjuvant chemotherapy for operable breast cancer. Br J Surg. 2007;94: 1189-200.

3. van der Hage JA, van de Velde CJ, Julien JP, Tubiana-Hulin M, Vandervelden C, Duchateau L. Preoperative chemotherapy in primary operable breast cancer: results from the European Organization for Research and Treatment of Cancer trial 10902. J Clin Oncol. 2001;19:4224-37.

4. Loo CE, Teertstra HJ, Rodenhuis S, van de Vijver MJ, Hannemann J, Muller SH, et al. Dynamic contrast-enhanced MRI for prediction of breast cancer response to neoadjuvant chemotherapy: initial results. AJR Am J Roentgenol. 2008;191:1331-8.

5. von Minckwitz G, Kummel S, Vogel P, Hanusch C, Eidtmann H, Hilfrich $\mathrm{J}$, et al. Neoadjuvant vinorelbine-capecitabine versus docetaxel-doxorubicin-cyclophosphamide in early nonresponsive breast cancer: phase III randomized GeparTrio trial. J Natl Cancer Inst. 2008;100:542-51.

6. Katz A, Saad ED, Porter P, Pusztai L. Primary systemic chemotherapy of invasive lobular carcinoma of the breast. Lancet Oncol. 2007;8:55-62.

7. Cocquyt VF, Blondeel PN, Depypere HT, Praet MM, Schelfhout VR, Silva OE, et al. Different responses to preoperative chemotherapy for invasive lobular and invasive ductal breast carcinoma. Eur J Surg Oncol. 2003;29:361-7.

8. Cristofanilli M, Gonzalez-Angulo A, Sneige N, Kau SW, Broglio $\mathrm{K}$, Theriault RL, et al. Invasive lobular carcinoma classic type: response to primary chemotherapy and survival outcomes. J Clin Oncol. 2005;23:41-8.

9. Tubiana-Hulin M, Stevens D, Lasry S, Guinebretière JM, Bouita $\mathrm{L}$, Cohen-Solal C, et al. Response to neoadjuvant chemotherapy in lobular and ductal breast carcinomas: a retrospective study on 860 patients from one institution. Ann Oncol. 2006;17:1228-33.

10. Sorlie T, Perou CM, Tibshirani R, Aas T, Geisler S, Johnsen H, et al. Gene expression patterns of breast carcinomas distinguish tumor subclasses with clinical implications. Proc Natl Acad Sci USA. 2001;98:10869-74.

11. Perou CM, Sorlie T, Eisen MB, van de Rijn M, Jeffrey SS, Rees $\mathrm{CA}$, et al. Molecular portraits of human breast tumours. Nature. 2000;406:747-52.

12. Nielsen TO, Hsu FD, Jensen K, Cheang M, Karaca G, Hu Z, et al. Immunohistochemical and clinical characterization of the basallike subtype of invasive breast carcinoma. Clin Cancer Res. 2004; 10:5367-74.

13. Hu Z, Fan C, Oh DS, Marron JS, He X, Qaqish BF, et al. The molecular portraits of breast tumors are conserved across microarray platforms. BMC Genomics. 2006;7:96.

14. Voduc D, Nielsen TO. Basal and triple-negative breast cancers: impact on clinical decision-making and novel therapeutic options. Clin Breast Cancer. 2008;8 Suppl 4:S171-S178.

15. Carey LA, Dees EC, Sawyer L, Gatti L, Moore DT, Collichio F, et al. The triple negative paradox: primary tumor chemosensitivity of breast cancer subtypes. Clin Cancer Res. 2007;13:232934.

16. Rouzier R, Perou CM, Symmans WF, Ibrahim N, Cristofanilli M, Anderson $\mathrm{K}$, et al. Breast cancer molecular subtypes respond differently to preoperative chemotherapy. Clin Cancer Res. 2005;11:5678-85.

17. Goldstein NS, Decker D, Severson D, Schell S, Vicini F, Margolis $\mathrm{J}$, et al. Molecular classification system identifies invasive breast carcinoma patients who are most likely and those who are least likely to achieve a complete pathologic response after neoadjuvant chemotherapy. Cancer. 2007;110:1687-96.

18. Straver ME, Glas AM, Hannemann J, Wesseling J, van de Vijver MJ, Rutgers EJ, et al. The 70-gene signature as a response 
predictor for neoadjuvant chemotherapy in breast cancer. Breast Cancer Res Treat. 2010;119(3):551-8.

19. Hannemann J, Oosterkamp HM, Bosch CA, Velds A, Wessels $\mathrm{LF}$, Loo $\mathrm{C}$, et al. Changes in gene expression associated with response to neoadjuvant chemotherapy in breast cancer. J Clin Oncol. 2005;23:3331-42.

20. Citron ML, Berry DA, Cirrincione C, Hudis C, Winer EP, Gradishar WJ, et al. Randomized trial of dose-dense versus conventionally scheduled and sequential versus concurrent combination chemotherapy as postoperative adjuvant treatment of node-positive primary breast cancer: first report of Intergroup Trial C9741/Cancer and Leukemia Group B Trial 9741. J Clin Oncol. 2003;21:1431-9.

21. Lebowitz PF, Eng-Wong J, Swain SM, Berman A, Merino MJ, Chow CK, et al. A phase II trial of neoadjuvant docetaxel and capecitabine for locally advanced breast cancer. Clin Cancer Res. 2004;10:6764-9.

22. O'Shaughnessy JA, Blum JL. Capecitabine/taxane combination therapy: evolving clinical utility in breast cancer. Clin Breast Cancer. 2006;7:42-50.

23. van Tienhoven G, Borger JH, Passchier DH, Hart AA, Rutgers EJ, van Dongen JA et al. The prognostic significance of the axillary apex biopsy in clinically operable breast cancer. Eur $J$ Cancer. 1995;31A:1965-8.

24. Mazouni C, Peintinger F, Wan-Kau S, Andre F, Gonzalez-Angulo AM, Symmans WF, et al. Residual ductal carcinoma in situ in patients with complete eradication of invasive breast cancer after neoadjuvant chemotherapy does not adversely affect patient outcome. J Clin Oncol. 2007;25:2650-5.

25. Rieber A, Brambs HJ, Gabelmann A, Heilmann V, Kreienberg R, Kuhn T. Breast MRI for monitoring response of primary breast cancer to neo-adjuvant chemotherapy. Eur Radiol. 2002;12: 1711-9.
26. Rosen EL, Blackwell KL, Baker JA, Soo MS, Bentley RC, Yu D, et al. Accuracy of MRI in the detection of residual breast cancer after neoadjuvant chemotherapy. AJR Am J Roentgenol. 2003; 181:1275-82.

27. Mathieu MC, Rouzier R, Llombart-Cussac A, Sideris L, Koscielny S, Travagli JP, et al. The poor responsiveness of infiltrating lobular breast carcinomas to neoadjuvant chemotherapy can be explained by their biological profile. Eur J Cancer. 2004;40: $342-51$.

28. Bonnefoi H, Potti A, Delorenzi M, Mauriac L, Campone M, Tubiana-Hulin M, et al. Validation of gene signatures that predict the response of breast cancer to neoadjuvant chemotherapy: a substudy of the EORTC 10994/BIG 00-01 clinical trial. Lancet Oncol. 2007;8:1071-8.

29. Symmans WF, Peintinger F, Hatzis C, Rajan R, Kuerer H, Valero $\mathrm{V}$, et al. Measurement of residual breast cancer burden to predict survival after neoadjuvant chemotherapy. J Clin Oncol. 2007;25: 4414-22.

30. Buzdar AU, Ibrahim NK, Francis D, Booser DJ, Thomas ES, Theriault RL, et al. Significantly higher pathologic complete remission rate after neoadjuvant therapy with trastuzumab, paclitaxel, and epirubicin chemotherapy: results of a randomized trial in human epidermal growth factor receptor 2-positive operable breast cancer. J Clin Oncol. 2005;23:3676-85.

31. Bhattacharyya M, Ryan D, Carpenter R, Vinnicombe S, Gallagher CJ. Using MRI to plan breast-conserving surgery following neoadjuvant chemotherapy for early breast cancer. $\mathrm{Br} J$ Cancer. 2008;98:289-93.

32. Boetes C, Mus RD, Holland R, Barentsz JO, Strijk SP, Wobbes T, et al. Breast tumors: comparative accuracy of MR imaging relative to mammography and US for demonstrating extent. Radiology. 1995;197:743-7. 